\title{
Model-based species delimitation: are coalescent species reproductively isolated?
}

\author{
Luke C. Campillo $^{1 *}$, Anthony J. Barley ${ }^{1}$, Robert C. Thomson ${ }^{1}$
}

${ }^{1}$ Department of Biology, University of Hawai'i, Honolulu, HI, 96822

*Corresponding Author: campillo@hawaii.edu

(1)

Keywords: Multispecies Coalescent; BPP; genetic distance; Drosophila speciation (1)

\section{ABSTRACT}

A large and growing fraction of systematists define species as independently evolving lineages that may be recognized by analyzing the population genetic history of alleles sampled from individuals belonging to those species. This has motivated the development of increasingly sophisticated statistical models rooted in the multispecies coalescent process. Specifically, these models allow for simultaneous estimation of the number of species present in a sample of individuals and the phylogenetic history of those species using only DNA sequence data from independent loci. These methods hold extraordinary promise for increasing the efficiency of species discovery, but require extensive validation to ensure that they are accurate and precise. Whether the species identified by these methods correspond to the species that would be recognized by alternative species recognition criteria (such as measurements of reproductive isolation) is currently an open question, and a subject of vigorous debate. Here we perform an empirical test of these methods by making use of a classic model system in the history of speciation research, flies of the genus Drosophila. Specifically, we use the uniquely comprehensive data on reproductive isolation that is available for this system, along with DNA sequence data, to ask whether Drosophila species inferred under the multispecies coalescent model correspond to those recognized by many decades of speciation research. We found that coalescent based and reproductive isolation based methods of inferring species boundaries are concordant for $77 \%$ of the species pairs. We explore and discuss potential explanations for these discrepancies. We also found that the amount of prezygotic isolation between two species is a strong predictor of the posterior probability of species boundaries based on DNA sequence data, regardless of whether the species pairs are sympatrically or allopatrically distributed. 


\section{INTRODUCTION}

33 Due to the complex nature of the processes that drive speciation, the development of an

34 operational species concept is difficult. For many years the Biological Species Concept (Mayr

35 1963), or some variation of it requiring high (but potentially incomplete) levels of reproductive

36 isolation between populations, was the standard (Coyne and Orr 2004). Starting in the late 1990s,

37 work echoing earlier evolutionary species concepts (Simpson 1951; Wiley 1978) led many

38 evolutionary biologists to recognize that species might best be recognized as "metapopulation

39 lineages" using any number of data types (de Queiroz 1998, 2007). Under this metapopulation

40 conceptualization of species, the boundary between independent lineages (i.e., species) is

41 demarcated by any number of criteria, which may or may not include reproductive isolation.

42 Despite these conceptual changes, systematists remain in need of an operational framework for

43 species delimitation. One option for placing the practice of species delimitation in a more

44 explicit framework is to use statistical models of evolutionary relationships among genetic loci.

45 One of the most widely used methods for doing so, BPP (Yang and Rannala 2014; Rannala and

46 Yang 2017), is a Bayesian method based on the multispecies coalescent model (MSC). BPP can

47 be employed to analyze variation in genealogies among many loci to identify independent

48 coalescent lineages as species (Leaché and Fujita, 2010; Rannala and Yang, 2003).

49 These methods hold promise for greatly increasing the pace and accuracy of species

50 description, although their utility depends on whether the lineages identified correspond to

51 biologically defensible (or meaningful) species and the precision of these estimates. A number of

52 studies have looked at the efficacy of the MSC for species tree inference (e.g., Fujita et al. 2012;

53 Reid et al. 2014; Barley et al. 2018), but the concordance between independent coalescent

54 lineages and species boundaries has been less well characterized in the literature. Relevant

55 studies generally take one of two forms: 1) species boundaries identified under the MSC are 
56 compared to those identified by alternative approaches, with concordance viewed as an

57 indication of accuracy (e.g., Camargo et al. 2012; Willis 2017); or 2) Data simulated under

58 known demographic scenarios are analyzed under the MSC and the results are used to identify

59 the relative over- or under-splitting of lineages by the model (Sukumaran and Knowles 2017;

60 Barley et al. 2018; Luo et al. 2018). Both types of studies frequently find that the MSC identifies

61 more lineages as species than some consider appropriate (Leaché et al. 2019). This discrepancy,

62 compounded by the lack of a fully objective species criterion, has led to an increasing wariness

63 that MSC delimitation may inflate the number of species, often beyond what many systematists

64 find realistic.

65 These studies have been useful for characterizing the statistical performance of the MSC,

66 but can be difficult to interpret in the context of real species radiations. On one hand, there is the

67 possibility that the MSC identified genuine species that were missed by earlier studies because

68 they are morphologically cryptic or otherwise difficult to differentiate. On the other hand, in

69 simulation studies, the accuracy of results relies on the generating model (i.e., the simulation)

70 being an accurate representation of biological reality. If important aspects of species biology are

71 missing from the generating model we may draw inaccurate conclusions merely because our

72 simulation study is overly simple relative to nature. The challenges associated with both of these

73 approaches might be averted by turning to an empirical system where extensive data on the

74 nature of species boundaries themselves are available. In such a system, we can examine the

75 correspondence between lineages identified as being independent under the MSC and other

76 criteria placed on species boundaries, such as reproductive isolation.

77 Here we make use of an empirical study system in which an extraordinarily rich

78 understanding of reproductive isolation has been established for many species pairs, the genus

79 Drosophila. We use this to compare species identification based on reproductive isolation with 
80 species delimitation under the MSC. Drosophila is an intensively studied model organism in

81 many disciplines, and arguably the most influential clade for understanding the consequences of

82 reproductive isolation as a mechanism of species formation and perpetuation. Much of this focus

83 stems from the fact that experimental lab crosses are possible between species, which provides

84 information on which species pairs show intrinsic reproductive isolation (lab crosses do not take

85 extrinsic factors into account) and to what degree that isolation is complete. Two classic papers

86 by Coyne and Orr $(1989,1997)$ synthesized data on reproductive isolation derived from

87 experimental crosses and allozyme electrophoretic distance, which they used as a proxy for time

88 since divergence by assuming a constant molecular clock. These data were used to produce

89 estimates for the amount of total reproductive isolation (the combined accumulation of pre- and

90 postzygotic isolation) and time (using genetic distance as a proxy) required for complete

91 speciation. Here, complete speciation refers to a relaxed version of Mayr's Biological Species

92 Concept (Mayr 1963) that allows for low levels of gene flow between closely related species (see

93 also Coyne and Orr 2004).

These studies provide insight into the nature of how species boundaries themselves

95 become established. A central finding from Coyne and Orr $(1989,1997)$, and the one we focus

96 on here, is that the amount of reproductive isolation increases with time since divergence.

97 Building off this idea, we examined several aspects of how species identified under the MSC

98 model matched those identified based on experimental quantification of reproductive isolation

99 and genetic distance. Specifically, we were interested in measuring the correlation between the

100 amount of reproductive isolation and genetic distance between two species, and the posterior

101 probability that those two species were identified as independent coalescent lineages. By

102 quantifying the relationship between reproductive isolation and coalescent lineages we stand to

103 gain a better understanding of the relationship between the biological processes that drive 
104 speciation and promising statistical approaches to species delimitation. It should also be noted

105 that most studies of reproductive isolation look at comparisons between species, whereas most 106 practitioners of MSC species delimitation think about comparisons within species (i.e., at the

107 population level). This study is explicitly concerned with delimitation of nominal species.

Assessing the accuracy of species delimitation remains difficult because speciation is a continuous process and species boundaries are not always discrete. However, utilizing information on reproductive isolation allows us to assess the performance of these methods in a more empirically grounded framework than is otherwise possible. With this in mind, the research

112 goals for this study were to: 1) examine the relationship between coalescent and reproductive

113 isolation based species delimitation; 2) quantify how varying levels of pre- and postzygotic

114 isolation in allopatric and sympatric species pairs affect our ability to identify species under the

$115 \mathrm{MSC}$; 3) better understand the utility of MSC methods to increase the accuracy and precision of 116 species recognition in the presence of partial, or complete, reproductive isolation.

\section{MATERIALS \& METHODS}

\section{Dataset Assembly}

120 The lists of species pairs studied by Coyne and Orr $(1989,1997)$ were combined to assemble a

121 set to be used in the present study $(\mathrm{n}=108$ pairwise comparisons). Values for pre- and

122 postzygotic isolation were compiled from Coyne and Orr's two studies $(1989,1997)$, and

123 updated based on isolation measures from a more recent study (Yukilevich 2012). Total

124 reproductive isolation ( $T$; Coyne and Orr 1989) was calculated using the equation: $T=$ Pre +

125 (1 - Pre ) $x$ Post. In keeping with previous work, if a species pair only had data on prezygotic

126 or postzygotic isolation, and that value was greater than 0.95 or 1.0 , respectively, we considered

$127 T$ to equal the measure for which there was data (Coyne and Orr 1997). Conversely, if a species 
128 pair only had data for prezygotic or postzygotic isolation, and that value was less than 0.95 or

1291.0 , respectively, those species pairs were excluded from downstream analysis. Data on allozyme

130 genetic distance (D) comes originally from Coyne and Orr $(1989,1997)$, but more recently

131 updated by Yukilevich (2012).

132 For the purpose of this study, we follow the species recognition thresholds proposed by

133 Coyne and Orr (1997). Specifically, they proposed minimum values on total reproductive

134 isolation $(\mathrm{T} \geq 0.903)$ and genetic distance (sympatric pairs: $\mathrm{D} \geq 0.04$; allopatric pairs: $\mathrm{D} \geq 0.54$ )

135 required for maintenance of species boundaries. While we recognize that the use of any

136 particular threshold may raise concerns, we opted to use these for two reasons. First, by using the

137 same metrics employed by previous studies, we are able to make a direct comparison between

138 species delimitation based on reproductive isolation and delimitation based on MSC models.

139 Second, because MSC species delimitation has many practical implications, we wanted to focus

140 on values that would likely be the most relevant and widely used in empirical studies

141 We assembled a set of genes $(n=8)$ that have been sequenced across a wide taxonomic

142 breadth within Drosophila (van der Linde and Houle 2008; Yang et al. 2012). We then

143 downloaded whatever sequence data was available from GenBank for this set of genes for all

144 species $(n=59)$ on the compiled list. Custom Python and R scripts (R Core Team 2013) were

145 used for data cleanup. Species pairs were then sorted into eleven species groups for analysis

146 (Coyne and Orr 1997). Here, "species group" refers to a term used in Drosophila literature to

147 reference a monophyletic set of closely-related species (see O'Grady and DeSalle 2018). While a

148 large amount of mitochondrial data is available for Drosophila, we chose to include only one

149 mitochondrial gene (Cytochrome c oxidase subunit II: COII) for each species group because we

150 expect the entire mitochondrial genome to share a single coalescent history. We limited the final

151 data matrix for each group to include only those genes that were missing sequence data for no 
152 more than two species. Because some intensively studied species have an abundance of sequence

153 data relative to the other species in their group (e.g., Drosophila subobscura has 137 COII

154 sequences available, while the other six species in the Obscura group combine for 30 available

155 sequences), we pruned sequences from overrepresented taxa to make the amount of data more

156 even across species. Specifically, for species that had $>10$ sequences at a given locus, we

157 randomly pruned sequences until the number that remained was equal to the next most densely

158 sampled species. Sequence data was aligned for each group using MUSCLE (Edgar 2004) and

159 converted to PHYLIP format using DendroPy 4.2.0 (Sukumaran and Holder 2010). These eleven

160 matrices, one for each species group, were analyzed independently in all downstream analyses.

161 To check for any difference in electrophoretic distance and genetic distances calculated from

162 DNA sequence data, we calculated Jukes-Cantor genetic distances across all species pairs for

163 COII in Geneious v 7.1.9 (Kearse et al. 2012) and then compared those to Nei’s D (Fig. S1;

164 Table S1).

\section{MSC Analysis}

167 After assembling the dataset, we performed MSC species delimitation using BPP v 3.2 (Yang

168 and Rannala 2014) on each of the 11 species groups separately. BPP uses reversible-jump

169 Markov Chain Monte Carlo (rjMCMC) to estimate a Bayesian posterior distribution for different

170 species delimitation models. We jointly estimated the species tree topology and assignment of

171 individuals into species (referred to as "analysis A11" in BPP). Under this model we define a

172 starting tree, where each named species in the group is a "population", and then use the rjMCMC

173 to target the posterior distribution of possible models that may merge combinations of these

174 populations into a smaller number of species. This approach does not allow single populations to

175 be split more finely, and so the existing taxonomy within each species group (i.e., named species 
176 from Coyne and Orr 1997) forms the upper limit on the total number of possible species in each

177 analysis. We specified a prior distribution that assigns equal probability for the number of

178 species (i.e., all populations merged into one species or a different species for each population)

179 and then divides that probability by the proportion of compatible labeled histories

180 (speciesmodelprior $=2$; Yang 2015). We also set a prior that allows $\Theta$ (population size

181 parameter) to vary among loci according to specified heredity multipliers (Hey and Nielsen

182 2004) to account for the differences in effective population size between nuclear and

183 mitochondrial loci (heredity $=2$; Yang and Rannala 2014).

Two central parameters of the MSC model are for population sizes $(\Theta)$ and species

goal was to set up a diffuse, but credible, prior distribution for both of these parameters. Here,

we constructed an empirical prior for $\Theta$ in Drosophila by using published estimates of this value,

188 if available, or published estimates of effective population size $\left(N_{e}\right)$ and mutation rate $(\mu)$ to used the online database TimeTree (www.timetree.org) to obtain an estimated root age for each

194 species group. Because each species group had different estimated root ages, there was a unique

195 combination of $\Theta$ and $\tau$ prior gamma distributions for each group. For both prior distributions,

196 we centered the mean of the gamma distribution on the empirical values, and set the variance of

197 the distribution to be wider than the distribution of all empirical values. We ran 204,000 MCMC

198 generations, discarding the first 4000 generations as burnin and then sampling every 20

199 generations until we reached 10,000 samples (BPP output files will be available on Dryad Digital 
Repository upon publication). We repeated each analysis two times, checking that the results

201

202

203

204

205

206

207

208

209

210

211

212

213

214

215

216

217

218

219

220

221

222

223 remained consistent across runs (Yang 2015).

The BPP manual (Yang 2015) explicitly states that there are no default priors for $\Theta$ and $\tau$, yet many studies employ a set of three prior gamma distributions originally used by Leaché and Fujita (2010) as if they were. Here we explored prior sensitivity by performing additional BPP runs on each dataset under this suite of priors (Leaché and Fujita 2010). For the sake of clarity, we will refer to the set of priors from Leaché and Fujita (2010) as empirically "uninformed" and our empirically derived priors as "informed". In total, we ran a BPP delimitation analysis on each of the eleven species groups under four unique prior settings (one informed, three uninformed) resulting in 44 independent BPP runs. Within the uninformed set of priors, we focus on those that most closely matched our prior expectations about the coalescent history for Drosophila (large ancestral population and shallow divergence among populations in this case). This should minimize differences between informed and uninformed priors, thereby supplying a realistic (and somewhat conservative) test of prior sensitivity. Full details for all prior settings can be found in supplementary material (Table S2), including $\Theta$ and $\tau$ priors for all groups and BPP runs. We extracted posterior $\Theta$ values from each MCMC output file using a custom Python script. To summarize the posterior distribution for $\tau$, we used the R package Phytools v 0.6-60 (Revell 2012) to read the sampled trees in the MCMC output into R and calculate the tree height of each (Python and R codes will be available on Dryad Digital Repository upon publication).

We visually inspected concordance between prior and posterior distributions of $\Theta$ and $\tau$ for the informed and uninformed priors. Additionally, we investigated whether the number of species delimited was sensitive to the alternative priors.

The A11 analysis in BPP estimates posterior probabilities for different delimitation models that may differ in number of species and the topology of the species phylogeny. Here, we 
224 are specifically interested in the marginal posterior probability for the splitting, or lumping, of

225 each particular species pair, rather than the marginal posterior probability of any one delimitation

226 model. Because species pairs can be split or lumped in different configurations, we calculated the

227 marginal posterior probability of independence between each species in a given pair. We refer to

228 this value as the Posterior Probability of Independent Lineages (PPIL). PPIL values are

229 practically, and philosophically, the same measure as the "speciation probability" described in

230 Leaché and Fujita (2010). The difference is that we calculate these values from analyses that

231 marginalize across both species delimitations and species tree topologies, whereas Leaché and

232 Fujita (2010) only marginalized across species delimitations due to limitations of the software at

233 that time which required the species tree be held constant (i.e., we do not employ a guide tree in

234 our analysis, as was done previously).

235 We calculated PPILs for all species pairs from Coyne and Orr $(1989,1997)$ where

236 sufficient sequence data was available to conduct the analysis, and for which information on

237 reproductive isolation was available $(n=108$ pairwise comparisons. Using PPIL values, we

238 compared the number of independent lineages identified by the MSC to those based on

239 reproductive isolation and genetic distance documented by Coyne and Orr $(1989,1997)$. The two

240 species in each pair were considered independent lineages if they had a high PPIL $(\geq 0.95)$ or

241 met the criteria for total isolation $(T \geq 0.903)$ and genetic distance $\left(\mathrm{D}_{\text {allo }} \geq 0.54 ; \mathrm{D}_{\text {symp }} \geq 0.04\right)$

242 from Coyne and Orr (1997).

244 Reproductive Isolation and Genetic Distance

245 Besides a cursory comparison in numbers of species delimited by either method, we were also

246 interested in how the components of speciation considered by Coyne and Orr $(1989,1997)$

247 related to PPIL values in our present study. To accomplish this, we selected among a series of 
generalized additive models (GAMs) to assess the impact of different predictor variables

249 (prezygotic isolation, postzygotic isolation, and genetic distance) on the PPIL for allopatrically

250 and sympatrically distributed species pairs. We chose not to include total reproductive isolation

251 in these models because it is a function of prezygotic and postzygotic isolation, which we already

252 account for independently. We elected to use a GAM because the model can flexibly capture the

253 impact of a predictor variable through a smoother function, allowing for both linear and non-

254 linear relationships. Because the dependent variable (i.e., PPIL) included 0 and 1, we performed

255 a logit transformation to normalize the data using the R package car (Fox and Weisberg 2011).

256 We then used the R package mgvc to construct and fit GAMs to the data (Wood 2011). We

257 calculated Akaike Information Criterion (Akaike 1974) values for each model and considered the

258 model with the smallest AIC to be the best fitting model. We further considered models with

$259 \Delta \mathrm{AIC} \leq 2$ from the best model to be similarly plausible (Burnham et al. 2002).

We analyzed two independent sets of GAMs to: 1) explore the differences between

261 allopatric and sympatric species pairs and 2) investigate the impact of predictor variables within

262 allopatric and sympatric species pairs separately. The first series of GAMs compared models

263 with one smoother function for all species pairs to models specifying separate smoothers for

264 allopatric and sympatric species pairs. Improved fit for the latter model would indicate that range

265 overlap between taxa differentially predicts PPIL values, and therefore these two types of species

266 pairs (allopatric and sympatric) should be modelled separately in downstream analyses.

267 After confirming two smoothers based on geography was a better model, we fit the

268 second series of GAMs on allopatric, sympatric, and closely related $(\mathrm{D} \leq 0.50)$ allopatric and

269 sympatric species pairs. For each set of species pairs, we fit a total of five GAMs, modelling the

270 three predictor variables listed previously (prezygotic isolation, postzygotic isolation, and genetic

271 distance) and two additive models (prezygotic isolation + genetic distance and postzygotic 
272 isolation + genetic distance). We fit the GAMs on taxa with low genetic distance $(\mathrm{D} \leq 0.5)$ to

273 maintain consistency with Coyne and Orr (1989), and because they suggested closely related

274 sympatric species pairs should show the strongest signature of reinforcement. In this context,

275 evidence for reinforcement's effect would be that the model for prezygotic isolation is the

276 strongest predictor of PPIL among closely related sympatric species, followed closely by a

277 model of postzygotic isolation. Specifically, because reinforcement acts to increase prezygotic

278 isolation in order to counteract disadvantageous hybridization, there must be some level of

279 postzygotic isolation.

Because data from species pairs are phylogenetically non-independent, we also explored

281 how this might impact our results. To do so, we extracted the maximum a posteriori tree from

282 each of the BPP analyses using phytools v 0.6-60 (Revell 2012), setting branch lengths to their

283 marginal posterior mean. We then assembled a reduced set of phylogenetically independent

284 species pairs (i.e., only including pairs that are connected by paths on the tree that do not

285 intersect with other pairs; see Felsenstein 2004, pg. 444). We then reran all the GAM analyses on

286 this reduced but phylogenetically independent dataset.

287 Lastly, we investigated how PPIL values may vary with respect to levels of pre- and

288 postzygotic isolation. To do this, we first constructed a reduced dataset that included all species

289 pairs with prezygotic $(n=100)$ or postzygotic $(n=74)$ isolation data. As outlined by Coyne and

290 Orr $(1989,1997)$, both of these values range from 0-1.0, with complete reproductive isolation

291 (pre- or postzygotic) equal to 1.0. We then performed an ANOVA to determine if PPIL differed

292 according to the levels of pre- and postzygotic isolation. Because the measures of postzygotic

293 isolation increased from 0 to 1.0 in 0.25 increments, we treated each level as a separate category

294 for the ANOVA. For prezygotic isolation, we binned the values based on the same levels (e.g., 0-

$2950.25,0.25-0.50$, etc.). Coyne and $\operatorname{Orr}(1989,1997)$ investigate what role Haldane’s Rule 
296 (Haldane 1922) may play in the speciation process and find it to be an important early step. We

297 checked whether it might also influence PPIL by assembling a reduced dataset containing only

298 those species pairs with $0.25-0.75$ postzygotic isolation $(n=32)$, because at these levels of

299 postzygotic isolation the pair could either be in line with Haldane's Rule (i.e., the heterogametic

300 sex, males in this case, are either sterile or infertile for both crosses) or not (i.e., a male and

301 female, or both females are sterile/inviable). Information on sterility/infertility was taken from

302 Yukilevich (2012). We manually categorized species pairs as either conforming to Haldane's

303 Rule or not and performed a one-way t-test to look for differences between both categories of

304 species pairs.

305

306 RESULTS

307 Genetic dataset assembly and analysis

308 The final dataset included a total of 543 sequences from 8 genes. We were able to obtain

309 sequence data for all species from one gene, COII, totaling 160 sequences for that gene. The

310 median number of genes per species group was three. The Bipectinata group had the greatest

311 gene diversity $(n=6)$, while the Buzzatii group was represented by only two genes. The mean

312 number of sequences compared between each species pair in each species group was 7.25,

313 ranging from an average of three sequences per pair in the Affinis group, up to 13.7 sequences

314 per pair in the Melanogaster group. Complete information on number of genes per group and loci

315 per species pair can be found in Supplementary Material (Table S3). The average genetic

316 distance across all species pairs was 0.82 , ranging from 0.026 (D. heteroneura - D. silvestris) to

3171.95 (recorded for multiple species pairs in the Obscura group). 


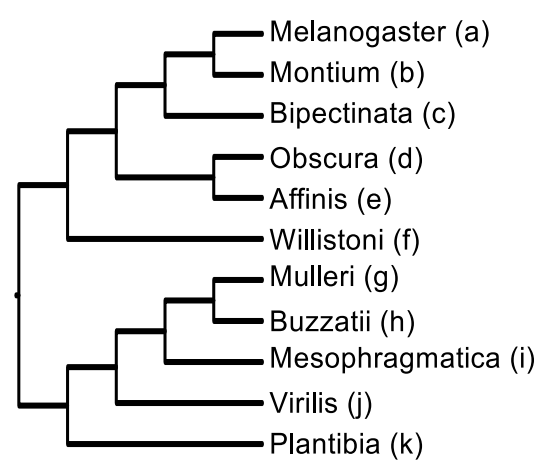

c) Bipectinata

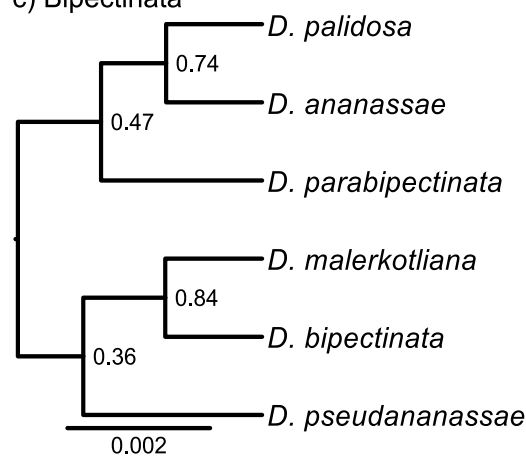

f) Willistoni

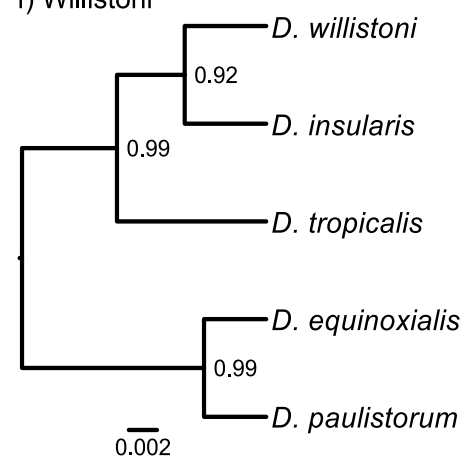

i) Mesophragmatica

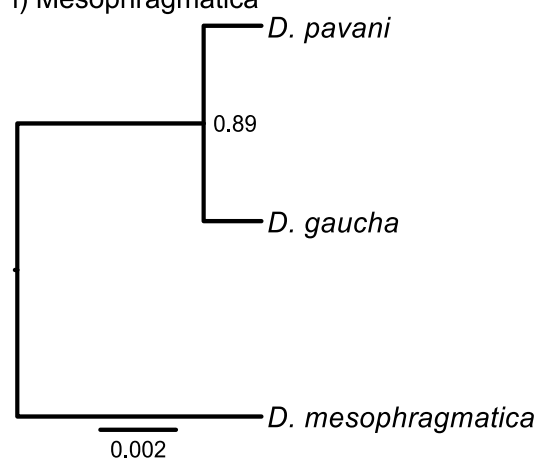

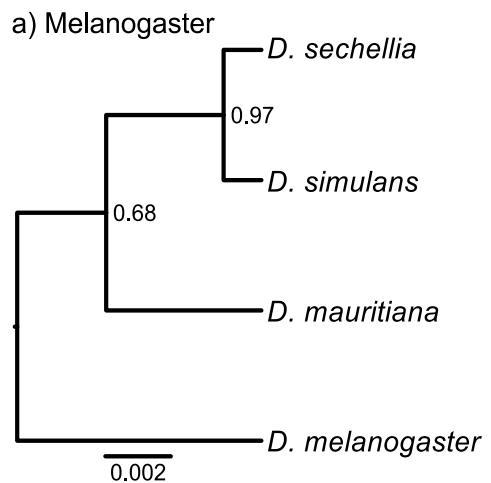

d) Obscura

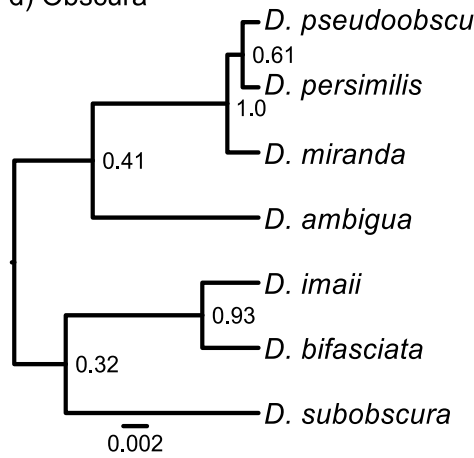

g) Mulleri

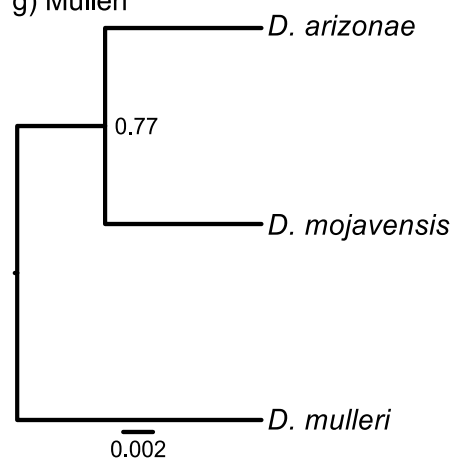

j) Virilis

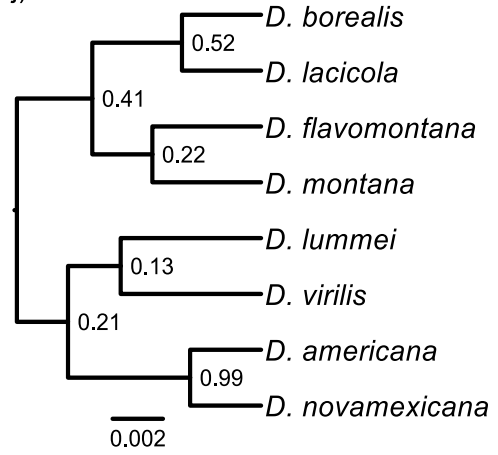

b) Montium

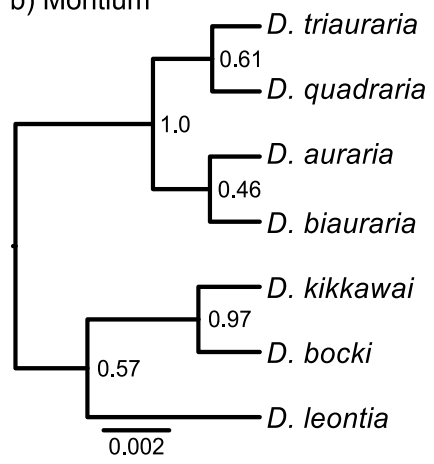

e) Affinis

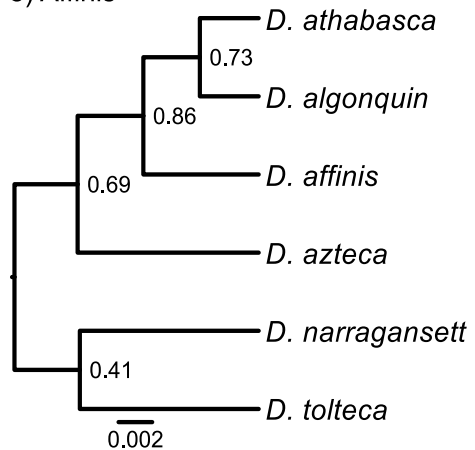

h) Buzzatii

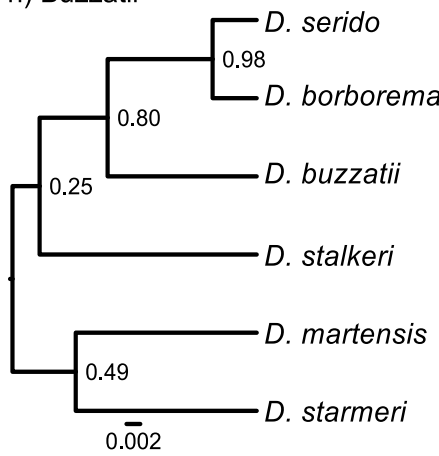

k) Plantibia

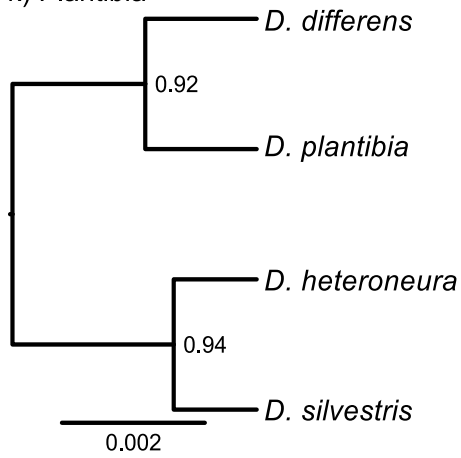

Figure 1) Consensus species trees for each species group in this study. Branch lengths (in coalescent units) are based on the average branch length from every MCMC tree that was topologically concordant with the highest probability species tree (taken from BPP output). Note that the scale bar is a different length for each tree because it represents the same value (0.002 coalescent units) for all trees. Backbone tree (top left) modified from Castillo (2017). 
We ran the BPP analyses two times for each species group, and obtained similar results

324 across runs. Consistency across runs indicates that mixing is likely adequate and the MCMC

325 chain did not get stuck on any one model (Yang 2015). Species trees for each species group

326 revealed varying levels of posterior probability support across nodes, but overall the species trees

327 correspond with results from other phylogenetic studies (Fig. 1; van der Linde and Houle 2008;

328 Yang et al. 2012).

\section{Concordance of reproductively isolated species and coalescent species delimitation}

331 The two approaches (i.e., threshold of genetic distance and reproductive isolation vs. PPIL threshold) recognized 63 out of the 99 species pairs as comprising two independent lineages. An additional 13 species pairs were not recognized as independent lineages by either approach. The

334 two approaches were therefore in agreement on the species status for 76 of 99 species pairs in 335 total ( 77\%; Fig. 2). Of the remaining 23 species pairs, 17 met the threshold for independent 336 lineages based on PPIL only, whereas the other six species pairs were considered to be distinct 337 species based on total reproductive isolation (T) and genetic distance (D) only. Qualitatively 338 similar, but somewhat more discordant, results were recovered when using the uninformed priors

339 (68\% agreement between methods). There was greater concordance between methods when 340 delimiting sympatric taxa than allopatric taxa (Fig. 2) and this pattern held true across the

341 informed and uninformed priors. When lowering the admittedly arbitrary, but common, PPIL

342 threshold from 0.95 to 0.85 we recovered roughly the same amount of concordance (75 out of 99

343 species pairs in agreement; $76 \%$ concordance). However, there were more lineages recognized 344 based on PPIL alone (Fig. S2). We also found greater discordance between methods for closely 345 related species pairs $(\mathrm{D} \leq 0.5)$ than those more distantly related $(\mathrm{D}>0.5$; Fig. $\mathrm{S} 3)$. 


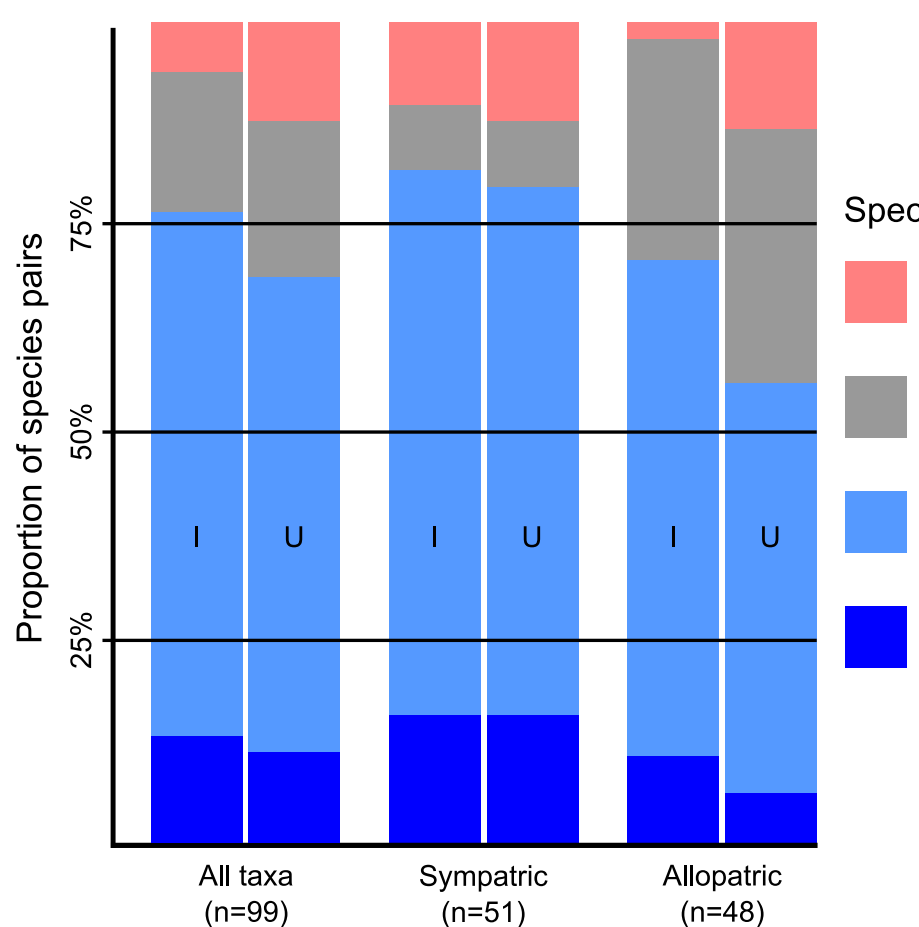

Species pairs delimited by: Reproductive isolation and genetic distance alone Multispecies coalescent model (PPIL value) alone Species pair split under both delimitation methods Species pair NOT split by either delimitation method

Figure 2) Plots comparing concordance between species delimitation models. Species delimitation models were in agreement for the majority of species pairs (Both; blue). However, there was discrepancy between certain species pairs. Some were split solely on reproductive isolation and genetic distance (RI + D; red) while others only on the basis of PPIL value $\geq 0.95$ (MSC; gray). Results are shown for both informed (I) and uninformed (U) prior settings.

\section{Reproductive Isolation and Genetic Distance}

We recovered better predictive models of PPIL when considering sympatric and allopatric taxa separately for prezygotic isolation and genetic distance, and similarly plausible models for postzygotic isolation (Table 1). When fitting the second set of GAMs to the different types of species pairs we found that the models of prezygotic isolation and genetic distance generally explained more null deviance than those for postzygotic isolation, which essentially reduced to a linear model (Fig. 3; plots for other GAM models are provided in Fig. S4 and Fig. S5). For allopatric taxa, the combined effect of prezygotic isolation + genetic distance was the best predictor of PPIL, but the effect of prezygotic isolation alone was similarly plausible $(\triangle \mathrm{AIC}=$ 1.11; Table 2). In sympatric taxa, prezygotic isolation was the best predictor of PPIL, followed closely by the similarly plausible combination model of prezygotic isolation + genetic distance $(\triangle \mathrm{AIC}=0.21 ;$ Table 2$)$. In the sympatric species pairs with low genetic distance $(\mathrm{D}<0.5)$, we 
364 found that the best predictor of PPIL was again prezygotic isolation, with models of genetic

365 distance and postzygotic isolation + genetic distance similarly plausible $(\triangle \mathrm{AIC}=0.66 \& \Delta \mathrm{AIC}$

$366=0.58$, respectively). We consistently found that the null deviance explained was low for the

367 postzygotic isolation models (Table 2). These results remained qualitatively unchanged for the

368 reduced phylogenetically independent datasets, with prezygotic isolation being the best predictor

369 of PPIL in allopatry and sympatry. We present the results from the full dataset here, while

370 complete results from the phylogenetically independent dataset can be found in the

371 Supplementary Material (Table S4).

372 When looking at species pairs with prezygotic isolation data $(n=100)$, we recovered a

373 significant difference between the level of prezygotic isolation and PPIL value, particularly

374 between species pairs with lower levels of prezygotic isolation (ANOVA; F-stat = 15.79; p-value

$375=2.01 \times 10^{-8}$; Fig. 4a). However, when considering all species pairs with postzygotic isolation

376 data $(n=74)$, we found that there was no statistical difference in PPIL among the five levels of

377 isolation $($ ANOVA; F-stat $=1.144 ;$ p-value $=0.343$; Fig. 4b). Additionally, although the mean

378 PPIL for species pairs that follow Haldane's Rule was lower $(0.927, \mathrm{n}=21)$ than those that do

$379 \operatorname{not}(0.97 ; \mathrm{n}=11)$, there was no statistically significant difference in PPIL between the groups (t-

380 test; $p$-value $=0.179 ;$ Fig. S6).

381 Table 1)

\begin{tabular}{ccccccc} 
& $\begin{array}{c}\text { Prez. Isolation } \\
(\mathbf{n}=\mathbf{1 0 0})\end{array}$ & $\begin{array}{c}\text { Post. Isolation } \\
(\mathbf{n = 7 4 )}\end{array}$ & $\begin{array}{c}\text { Gen. Distance } \\
(\mathbf{n}=\mathbf{1 0 8})\end{array}$ \\
\cline { 2 - 7 } Number of Smoothers & $\Delta$ AIC & $\begin{array}{c}\text { Dev. } \\
\text { Exp. }\end{array}$ & $\Delta$ AIC & $\begin{array}{c}\text { Dev. } \\
\text { Exp. }\end{array}$ & $\Delta$ AIC & $\begin{array}{c}\text { Dev. } \\
\text { Exp. }\end{array}$ \\
\hline One (Allo/Symp Together) & 8.15 & 0.29 & $* * *$ & 0.07 & $1.85^{\dagger}$ & 0.22 \\
Two (Allo/Symp Separate) & $* * *$ & 0.40 & $1.94^{\dagger}$ & 0.07 & $* * *$ & 0.27
\end{tabular}

Table 1) Results from a series of GAMs to test if relationships are different between allopatric and sympatric species pairs, where $* * *$ means that model had the lowest AIC value and is therefore the most plausible model. The dagger $(\dagger)$ indicates a similarly plausible model $(\Delta \mathrm{AIC} \leq 2$; Burnham et al. 2002). The proportion of null deviance explained by the model ("Dev. Exp.") is also provided. 


\section{Effect of the Priors}

388 We did not see an effect of the informed versus uninformed priors on the mean number of 389 lineages delimited in a given group (i.e., BPP delimited a consistent number of species in each group across different prior settings). However, we did see a difference in the variance (i.e., the

391 prior influenced how much uncertainty there was in the number of species in each group; Fig. are a relatively poor match for Drosophila, which has the effect of biasing the results to be accurately estimating the variance of a random variable, in addition to accurately estimating its mean.

Table 2)

401

402

403

404

405

406

407

408

409

\section{Explanatory Variable}

\section{Prezygotic Isolation}

Postzygotic Isolation

Genetic Distance

Pre. Isol. + Gen. Dist.

Post. Isol. + Gen. Dist.

\section{Allopatric}

(n= 28)

$\triangle \mathrm{AIC}$

$1.11^{\dagger}$

14.80

11.19

Dev.

Exp.

Allopatric $(\mathrm{D} \leq \mathbf{0 . 5 0} ; \mathrm{n}=15)$

0.51

$\triangle \mathrm{AIC}$

\begin{tabular}{c} 
Dev. \\
Exp. \\
\hline 0.61 \\
0.07 \\
0.34 \\
0.40 \\
0.40
\end{tabular}

\section{Sympatric}

$(n=42)$

$\triangle \mathrm{AIC}$

\begin{tabular}{cc}
\hline$* * *$ & 0.21 \\
\hline $1.25^{\dagger}$ & 0.07 \\
2.44 & 0.05 \\
$0.21^{\dagger}$ & 0.23 \\
2.88 & 0.08
\end{tabular}

Sympatric $(\mathrm{D} \leq \mathbf{0 . 5 0} ; \mathbf{n}=\mathbf{2 3})$

Table 2) Table of $\triangle \mathrm{AIC}$ values, where $* * *$ indicates the most plausible model (i.e., which explanatory variable best explains PPIL value). The dagger $(\dagger)$ indicates a similarly plausible model ( $\triangle \mathrm{AIC} \leq 2$; Burnham et al. 2002$)$. The plus sign $(+)$ between variables indicates a model of the additive effect of two predictors. The proportion of null deviance explained by the model ("Dev. Exp.") is also provided.

\section{DISCUSSION}

\section{Concordance of Species Boundaries Under the MSC}

We assessed concordance between species that are delimited under population genetic models of 
411 distinctiveness, reproductive isolation. Specifically, we looked at species delimitations inferred

412 under the BPP implementation of the MSC (which we consider an operational framework for a

413 lineage-based species concept; Simpson 1951; Wiley 1978; De Queiroz 2007), and those

414 considered distinct species determined by the amount of reproductive isolation and genetic

415 distance between two lineages (i.e., a modified Biological Species Concept; Mayr 1963; Coyne 416 and Orr 2004).

417 We find that the methods do not return identical results, and the multispecies coalescent

418 tends to more readily split species than measures of reproductive isolation and genetic distance

419 alone. This could result from the MSC recognizing genuine species that might be missed by

420 other approaches. Specifically, our results confirm that (if we take MSC delimitation as truth)

421 reproductive isolation does not need to be complete in order for lineages to be identifiable as

422 independent. Alternatively, recent studies have found the MSC may be prone to oversplitting and

423 might result in delimiting “population structure, not species” (Sukumaran and Knowles 2017;

424 Chambers and Hillis 2019). However, because we are attempting to delimit nominal species any

425 population structure within species should not mislead our interpretations of the species

426 delimitation models. That being said, this also means that our results may provide a somewhat

427 optimistic view of MSC performance.

428 When using the PPIL $\geq 0.95$ cutoff, both criteria agreed with one another in the majority,

429 but far from all, cases (76\% agreement; PPIL values for all species pairs found in Table S5). Of

430 these species pairs, 63 were delimited by both methods and 13 were delimited by neither. The

431 cases where neither delimitation model recognizes these nominal taxa as independent lineages

432 represent scenarios in which the taxonomy may require reexamination. For example, none of the

433 species pairs in the Auraria species complex were recognized as independent by either method,

434 suggesting current taxonomy in this species complex may be recognizing too many species. 
Watada et al. (2011) recently took one step in this direction, revising the taxonomy of $D$.

quadraria and suggesting it is better categorized as a junior synonym of $D$. triauraria.

Of the remaining species pairs $(n=23)$ for which there is disagreement between methods,

438 the majority $(n=17)$ were pairs split under the MSC (i.e., high PPIL) but not under the Coyne and

439 Orr criteria. Most of these high PPIL pairs did not have enough total reproductive isolation $(\mathrm{n}=$

440 14) to meet the Coyne and Orr threshold, while the remaining three species pairs did not have

441 great enough genetic distance. These three species pairs are all allopatrically distributed. Due to

442 their high levels of reproductive isolation (between 0.94-1.0), we expect that they would remain

443 distinct upon secondary contact.

Of the 14 species pairs failing to meet the minimum total reproductive isolation

446 do not take geographic or environmental reproductive isolation into account, it is possible that

447 allopatric pairs have not evolved a high degree of pre- or postzygotic isolation simply because

448 they are genetically isolated by virtue of their distribution (i.e., geographic isolation is a strong

449 barrier to gene flow, in itself). For example, the allopatric species pair of D. americana and $D$.

450 virilis had relatively low genetic distance $(\mathrm{D}=0.54)$ and total isolation $(\mathrm{T}=0.644)$, but were

451 never lumped under the MSC model $(\mathrm{PPIL}=1.0)$. Furthermore, detailed crossing experiments

452 and QTL mapping have shown high levels of postmating, prezygotic isolation between these two

453 species (Sweigart 2010), suggesting that genetic distance and total reproductive isolation may

454 take more time to evolve relative to isolation identified under a coalescent framework. The other

455 four species pairs with high PPIL but low reproductive isolation were all sympatric. These may

456 represent cases where BPP is identifying incipient species with (potentially) low levels of

457 ongoing or recent gene flow (Leaché et al. 2019), or cases where the amount of reproductive

458 isolation may have been underestimated. For example, the sympatric species pair D. lummei-D. 
459 virilis would be considered independent lineages under the MSC (PPIL = 1.0) despite having the

460 lowest total isolation value in the entire dataset $(\mathrm{T}=0.263)$. However, at least one source

461 (Heikkinen and Lumme 1991) reported high postzygotic isolation between the pair that was not

462 reflected in either Coyne \& Orr $(1989,1997)$ or Yukilevich $(2012)$, and therefore was not

463 included our calculation of total isolation for this species pair.

464 The remaining six species pairs in disagreement would be considered species based on

465 reproductive isolation and genetic distance, but were not identified as independent lineages under

466 the MSC. At face value, this would indicate these are reproductively isolated species pairs that

467 cannot be identified as independently coalescing lineages. Of these, five are sympatric, and only

468 one is allopatric. For the only allopatric pair (D. mesophragmatic - D. pavani), the PPIL value

469 was barely below the 0.95 posterior probability cutoff $(\mathrm{PPIL}=0.942)$, and probably represents a

470 valid split under both methods. We recovered a range of PPIL values for the five sympatric

471 species pairs (PPIL between 0.794-0.930). Additionally, we observed that for all the sympatric

472 species pairs with high reproductive isolation but PPIL $\leq 0.95$ one or both species in that pair

473 have documented chromosomal inversions (Jha and Rahman 1973; Johnson 1985; Noor et al.

474 2001).

475 In principle, any of these discrepancies could be attributed to error in measures of

476 reproductive isolation, genetic distance, or PPIL (e.g., poor fit of the MSC to the data).

477 Widespread error in reproductive isolation measurements seems unlikely due to the controlled

478 laboratory conditions under which these data were collected. However, extrinsic isolating

479 mechanisms (e.g., ecological selection against hybrids) are not considered in a laboratory

480 environment, and may represent a greater source of discordance between laboratory and nature

481 than anything else. The amount of genetic distance could also have been overestimated or

482 underestimated, but this seems unlikely to be a systematic issue given that we found a strong 
483 correlation between Nei's electrophoretic distance and Jukes-Cantor genetic distance based on

484 COII (Fig. S1). While it could be true that species pairs are not as distinct as the amount of

485 genetic distance and reproductive isolation between them might suggest, it is also possible that

486 we failed to delimit them due to a lack of statistical power, driven by a paucity of loci used to fit

487 the MSC model. The lowest PPIL among reproductively isolated species (D. algonquin $-D$.

488 athabasca; PPIL = 0.79) was based on sequence data from only one individual per species for

489 two genes, which is among the lowest amount of data for any species pair in the entire dataset

490 (Table S3). Discordance between methods in these cases may simply be driven by the amount of

491 data available for analysis, rather than a true disagreement between methods. However,

492 performance in BPP is good even with a small number of loci (Rannala and Yang 2017), and we

493 found no relationship between the number of loci compared between species pairs and the PPIL

494 value for that species pair (linear regression: $\mathrm{R}^{2}=0.002$; $\mathrm{p}$-value $=0.61$; Fig. S7).

\section{Reproductive Isolation and GAMs}

497 We observed significant model improvement when specifying separate smoothers for sympatric

498 and allopatric taxa, rather than considering all taxa jointly, for most explanatory variables (Table

499 1). Based on these results, we subsequently explored the predictive power of three variables, and 500 two combinations of reproductive isolation and genetic distance (see Materials \& Methods), to

501 predict PPIL values for sympatric and allopatric taxa separately. In allopatric taxa, we found that 502 the combination model of prezygotic isolation + genetic distance best predicted PPIL value, with 

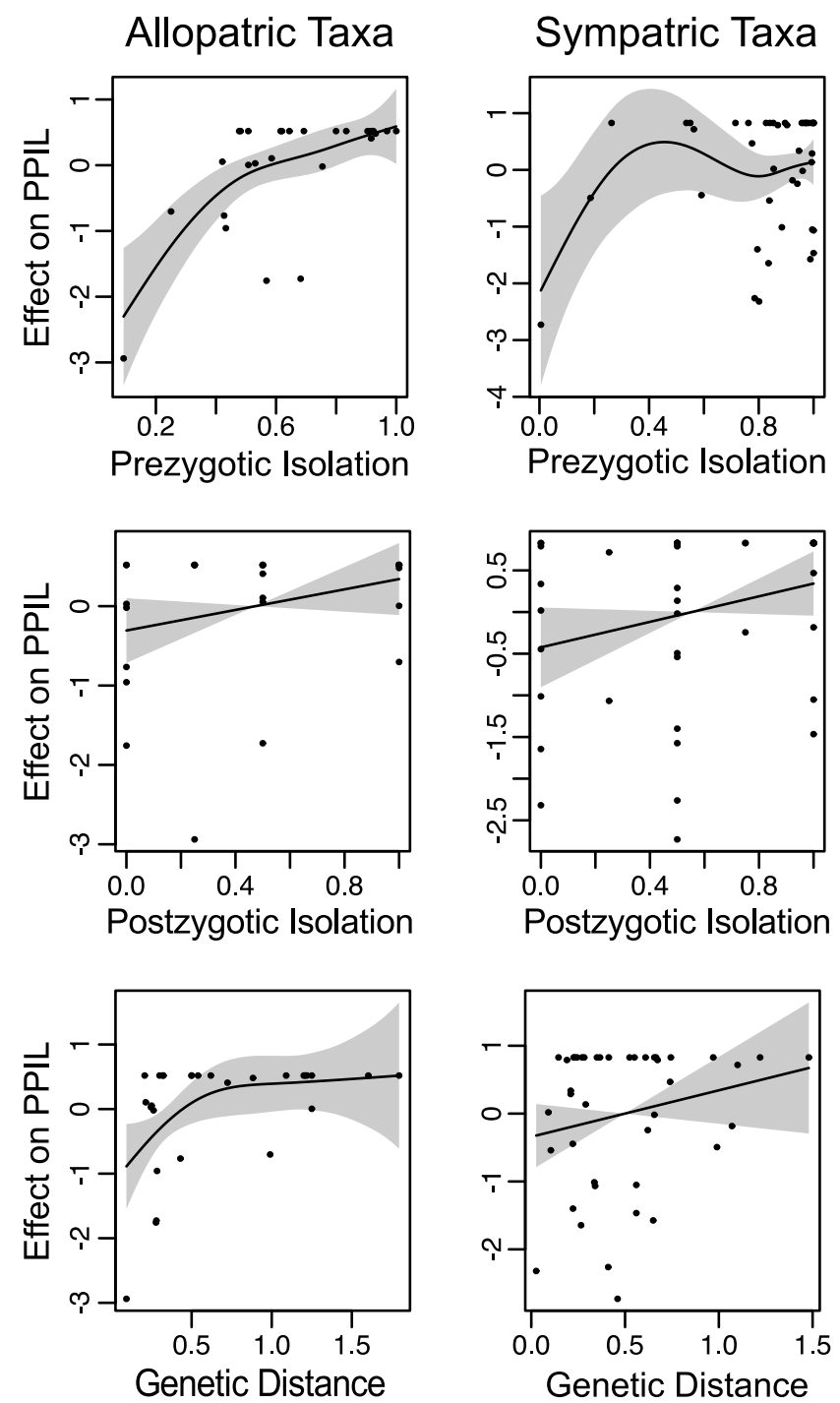

Figure 3) Fitted GAMs for allopatric (left) and sympatric (right) taxa. Note that the smooths are centered on zero to ensure model identifiability, meaning the y-axis is scaled relative to PPIL values but is not on the same scale (i.e., not from $0-1.0)$.

prezygotic isolation alone being found to be similarly plausible $(\triangle \mathrm{AIC}=1.11)$. This indicates

that knowledge about prezygotic isolation, particularly in conjunction with genetic distance, is a

strong predictor of PPIL values for allopatric species pairs. Although this makes sense at face

value, it is interesting that prezygotic isolation represents a good predictor of speciation for taxa

whose ranges do not overlap and therefore do not have the potential to mate in nature (i.e.,

prezygotic isolation does not matter if taxa are allopatrically distributed). Furthermore, the fact 
515 that the model of postzygotic isolation was the worst predictor of PPIL for allopatric species

516 pairs $(\triangle \mathrm{AIC}=14.8)$ suggests the fate of hybrids between allopatric taxa, either through lab trials

517 or secondary contact, says little about the independence of those lineages under the MSC.

518 Models of genetic distance and postzygotic isolation + genetic distance were also poor predictors

519 of PPIL in allopatric taxa. Taken together, this seems to imply that neither the time since two

520 species became geographically isolated (inferred from genetic distance) nor levels of hybrid

521 sterility/inviability are particularly indicative of lineage independence in allopatric taxa.

523 than all other explanatory variables, although the combination model of prezygotic isolation +

524 genetic distance represented a similarly plausible alternative model $(\triangle \mathrm{AIC}=0.206)$. This agrees

525 with the expectation that prezygotic isolation should be important in maintaining species

526 boundaries in sympatry. When looking at sympatric taxa at low genetic distance $(\mathrm{D}<0.5)$, we

527 found the combined model of prezygotic isolation + genetic distance model best predicted PPIL.

528 This suggests that time since divergence (inferred from genetic distance), and not just the amount

529 of prezygotic isolation, is an important indicator of lineage independence among closely related

530 and recently diverged sympatric taxa. The model for postzygotic isolation was the worst

531 predictor of PPIL for closely related sympatric taxa $(\triangle \mathrm{AIC}=4.56)$. Furthermore, in contrast to

532 the finding from Coyne and Orr $(1989,1997)$ we find that prezygotic isolation is the best

533 predictor of PPIL in closely related $(\mathrm{D} \leq 0.50)$ allopatric and sympatric species pairs. Given that

534 reinforcement, by definition, does not occur in allopatry (i.e., there are no unfit hybrids), this

535 reaffirms the relative importance of prezygotic isolation throughout the speciation process, even

536 in the absence of any postzygotic isolation.

ANOVA tests revealed a significant difference between the level of prezygotic isolation

538 and PPIL, but not between level of postzygotic isolation and PPIL, which recapitulates the 
539 results of the GAM analysis. Furthermore, this shows that once a low level of prezygotic

540 isolation has been obtained $(\geq 0.25)$ there is no statistical difference in PPIL values at higher

541 levels of prezygotic isolation. Lastly, here was no statistically significant difference in PPIL for

542 partially reproductively isolated species pairs (postzygotic isolation between $0.25-0.75$ ) that do

543 or do not show signature of Haldane's Rule. Although there is abundant support for the

544 occurrence of Haldane's Rule in nature (Delph and Demuth 2016), sterility/infertility of the

545 heterogametic sex does not seem to serve as a strong predictor of whether two lineages are

546 identified as independent coalescent lineages.

\section{Effect of the Prior}

549 Overall, we observed relatively consistent PPIL values across all prior settings (Table S4),

550 indicating results from BPP analyses are not especially sensitive to the prior for either $\Theta$ or $\tau$. As

551 we would expect, we observed larger differences between the prior and posterior distribution of

$552 \Theta$ and $\tau$ for the uninformed priors than the informed priors (Fig. 4a). The informed priors

553 allowed the MCMC to more widely explore topological space, both in terms of the number of

554 species in a given topology and the frequency of proposing a unique topology. Uniformed priors

555 are likely to depart strongly from empirically reasonable values for any particular dataset, which

556 can constrain how well the MCMC explores areas of parameters space that may have high

557 likelihoods (but very low prior probability). While we did not observe strong impacts on the

558 mean number of species that were inferred for these data, the misspecified priors did lead to

559 inflated estimates of certainty (Fig. 4b). Given the existing concerns regarding whether the MSC

560 tends to oversplit lineages, an exaggerated increase in precision, and presumably accuracy in

561 certain situations, would almost certainly exacerbate any innate complications of MSC species

562 delimitation (see also Leaché et al. 2019 for an alternative approach to better characterizing

563 uncertainty in MSC species delimitation). 
a)

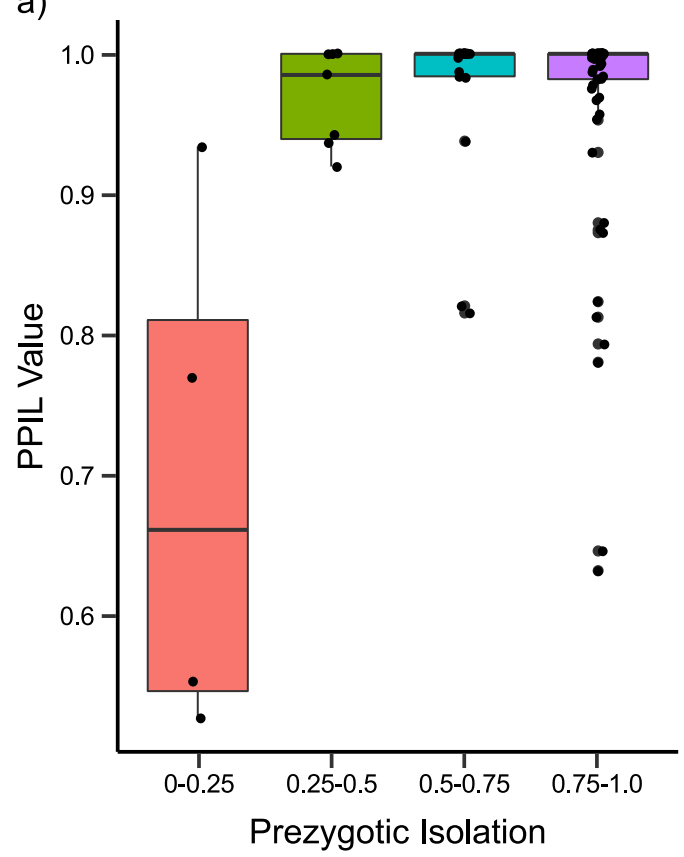

b)

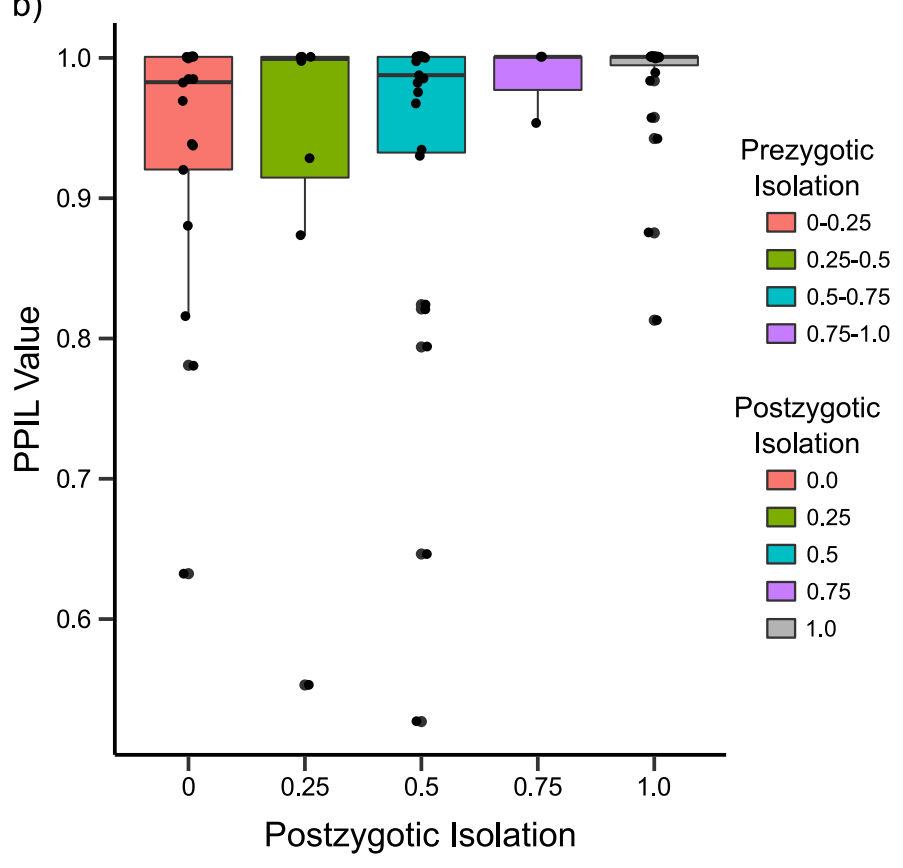

565

Figure 4) Boxplots depicting the results of ANOVA test across different levels of pre- or postzygotic isolation. There was a significant difference for prezygotic isolation $\left(\mathrm{p}\right.$-value $=2.01 \times 10^{-8}$ ), but not for postzygotic isolation $(\mathrm{p}$-value $=0.343)$.

573 Implications for the practice of species delimitation

574 then any model of species delimitation should recover them. Moreover, while the frequency of

575 speciation-collapse in nature is not yet well known, recent work has led to an increased recognition that the evolutionary history of many species is reticulate in nature (i.e., periods of

577 gene flow during and/or after speciation; Burbrink and Gehara 2018; Marques et al. 2019) or

578 otherwise non-bifurcating (e.g., budding or anagenic speciation; Silvestro et al. 2018). Taken

579 together, our results confirm that (if we take MSC delimitation as truth) reproductive isolation 
Alternatively, the MSC model may not be able to pick up on reproductive isolating mechanisms that are specific to particular regions of the genome (i.e., genomic islands of divergence; Wolf and Ellegren 2016). In this study, we found that for sympatric species with

584 high reproductive isolation, but low PPIL value, chromosomal rearrangement could be

585 implicated in the maintenance of species boundaries. Moreover, while chromosomal

586 rearrangements have long been associated with reproductive isolation and speciation (Rieseberg

587 2001; Campbell et al. 2018), to our knowledge have not been formally investigated under a MSC

588 model. Supposing chromosomal inversions drive a rapid increase in reproductive isolation, the

589 effects of localized genomic islands of divergence may not extend throughout the genome or be

590 reflected in the few loci being used for delimitation. Although selecting a number of genes from

591 a heterogeneous gene pool will often provide a useful approximation of species limits, if the only

592 difference between species are relatively small genomic islands of divergence (e.g.,

593 chromosomal inversions) the results from MSC delimitation may be inconsistent with the levels

594 of reproductive isolated observed in nature.

This study also allowed us to reveal novel insight into the speciation process. Most

596 notably, we found that levels of prezygotic isolation were consistently related to PPIL, and found

597 no indication that postzygotic isolation was similarly informative. Although quantification of

598 pre- and postzygotic isolation are not readily available outside of model organisms, the

599 implications of this finding potentially extends beyond the lab. Specifically, they may indicate

600 that prezygotic isolation evolves earlier in the speciation process and rapidly leads to identifiable

601 independent lineages, even under a relatively small amount of assortative mating (prezygotic

602 isolation $\geq 0.25$ ). This may provide some insight into why hybridization appears to be more

603 common in nature than was previously appreciated (Taylor and Larson 2019). As long as most of

604 the gene flow is within species, the boundaries are maintained and the speciation process in not 
a)
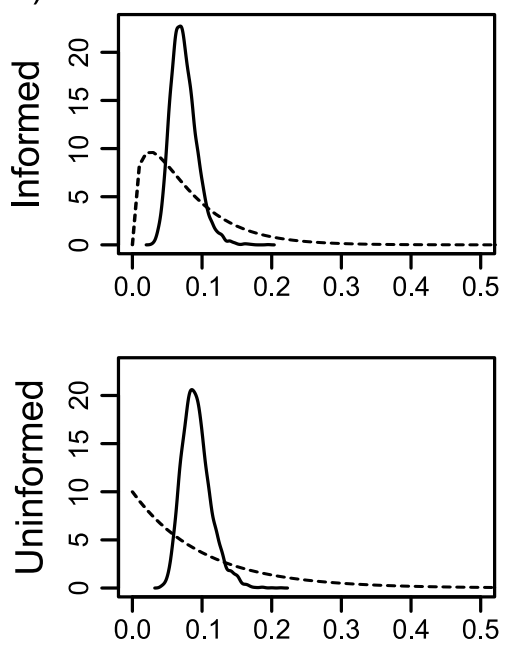

$\theta$
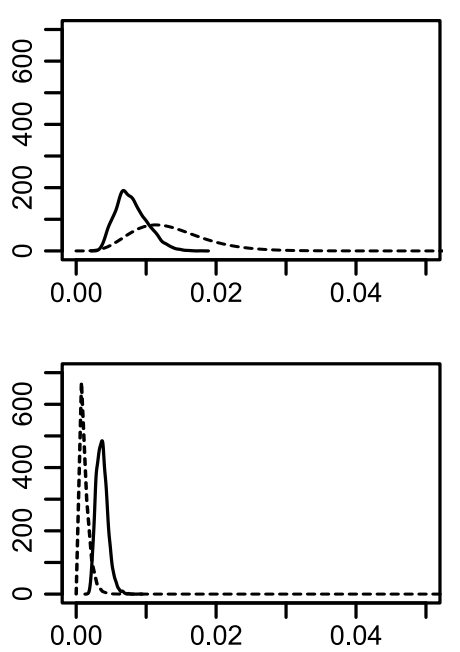

$\tau$ b)

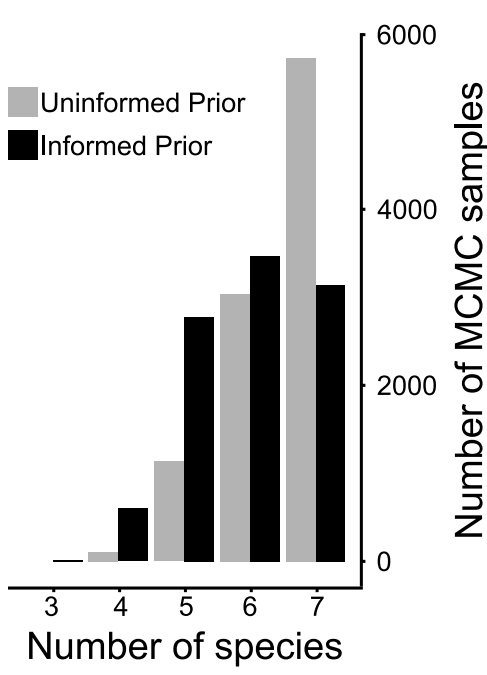

Figure 5a) This figure compares relationships between prior (dashed) and posterior (solid) gamma distributions of $\Theta$ and $\tau$ using informed (top) and uninformed (bottom) prior distributions for the Montium group. Plots for all other species groups can be found in the Supplementary Material (Fig. S8). b) Shows the difference between the number of species delimited under unformed (gray) and uninformed (black) prior setting out of the total 10,000 MCMC samples per run.

\section{CONCLUSION}

While reproductive isolation has long been a cornerstone of speciation research, it can rarely be used as an operational delimitation method because collecting such data is not tractable for most systems. Likewise, many practitioners of model-based species delimitation do not take reproductive isolation into account because the data are unavailable. In this study, we focus on a

621 Drosophila dataset that is uniquely suited to address this divide in how species are understood.

622 Here, we formally investigated how species boundaries based on reproductive isolation compare 623 to model based species delimitation. We found that model based and reproductive isolation based 
625 pairs $(\sim 17 \%)$, while $6 \%$ of the species pairs were delimited based on reproductive isolation and

626 genetic distance alone. However, because our study design does not allow for within species

627 population structure to be misinterpreted as species boundaries, our results may provide a

628 somewhat optimistic view of MSC performance. Additionally, by using a set of informed and

629 uninformed prior settings we were able to qualitatively assess the accuracy and precision of MSC

630 species delimitation, as implemented by BPP. In accordance with earlier studies, BPP appears to

631 be relatively robust to prior misspecification, although we do find evidence that prior

632 misspecification can lead to overconfidence in estimates of species boundaries. Lastly, based on

633 predictive GAM models, we found that the amount of postzygotic isolation between species pairs

634 was relatively uninformative as to whether or not those lineages can be recognized as

635 independent under the coalescent. We are not questioning that postzygotic isolation acts as a

636 strong reproductive barrier, certainly it does. However, it seems that prezygotic isolation

637 evolving early in the speciation process is closely tied to recognizably independent lineages. As

638 such, prezygotic isolation may be relatively more important in the incipient stages of speciation, 639 and possibly to the process as a whole.

\section{SUPPLEMENTARY MATERIAL}

642 Data available from the Dryad Digital Repository: XXXXXX

\section{FUNDING}

645 This work was supported by the US National Science Foundation (grant numbers DEB 1354506,

646 DEB 1754350, and DBI 1356796). This work was also supported by computational resources

647 provided by the University of Hawai'i Cyberinfrastructure group, as well as funding from the

648 Arnold and Mabel Beckman Foundation. 


\section{REFERENCES}

Akaike H. 1974. A new look at the statistical model identification. IEEE Transactions on Automatic Control. 19:716-723.

Barley A.J., Brown J.M., Thomson R.C. 2018. Impact of Model Violations on the Inference of Species Boundaries Under the Multispecies Coalescent. Systematic Biology. 67:269-284.

Burbrink F.T., Gehara M. 2018. The Biogeography of Deep Time Phylogenetic Reticulation. Systematic Biology. 67:743-755.

Burnham K.P., Anderson D.R., Burnham K.P. 2002. Model selection and multimodel inference: a practical information-theoretic approach. New York: Springer.

Camargo A., Morando M., Avila L.J., Sites J.W. 2012. Species delimitation with ABC and other coalescent-based methods: a test of accuracy with simulations and an empirical example with lizards of the Liolaemus darwinii complex (Squamata: Liolaemidae): species delimitation with ABC. Evolution. 66:2834-2849.

Campbell C.R., Poelstra J.W., Yoder A.D. 2018. What is Speciation Genomics? The roles of ecology, gene flow, and genomic architecture in the formation of species. Biological Journal of the Linnean Society. 124:561-583.

Castillo D.M. 2017. Factors contributing to the accumulation of reproductive isolation: A mixed model approach. Ecology and Evolution. 7:5808-5820.

Chambers E.A., Hillis D.M. 2019. The Multispecies Coalescent Over-Splits Species in the Case of Geographically Widespread Taxa. Systematic Biology.

Charlesworth B. 2009. Fundamental concepts in genetics: Effective population size and patterns of molecular evolution and variation. Nature Reviews Genetics. 10:195-205.

Coyne J.A., Orr H.A. 1989. Patterns of Speciation in Drosophila. Evolution. 43:362.

Coyne J.A., Orr H.A. 1997. "Patterns of Speciation in Drosophila" Revisited. Evolution. 51:295.

Coyne J.A., Orr H.A. 2004. Speciation. Sunderland, Mass: Sinauer Associates.

Cutter A.D. 2008. Divergence Times in Caenorhabditis and Drosophila Inferred from Direct Estimates of the Neutral Mutation Rate. Molecular Biology and Evolution. 25:778-786.

Delph L.F., Demuth J.P. 2016. Haldane's Rule: Genetic Bases and Their Empirical Support. Journal of Heredity. 107:383-391.

Edgar R.C. 2004. MUSCLE: multiple sequence alignment with high accuracy and high throughput. Nucleic Acids Research. 32:1792-1797.

Eyre-Walker A., Keightley P.D., Smith N.G.C., Gaffney D. 2002. Quantifying the slightly deleterious mutation model of molecular evolution. Mol. Biol. Evol. 19:2142-2149.

Felsenstein J. 2004. Inferring phylogenies. Sunderland, Mass: Sinauer Associates.

Fox J., Weisberg S. 2011. An R Companion to Applied Regression. Thousand Oaks CA: Sage.

Fujita M.K., Leaché A.D., Burbrink F.T., McGuire J.A., Moritz C. 2012. Coalescent-based species delimitation in an integrative taxonomy. Trends in Ecology \& Evolution. 27:480 488.

Haag-Liautard C., Dorris M., Maside X., Macaskill S., Halligan D.L., Charlesworth B., Keightley P.D. 2007. Direct estimation of per nucleotide and genomic deleterious mutation rates in Drosophila. Nature. 445:82-85.

Haldane J.B.S. 1922. Sex ratio and unisexual sterility in hybrid animals. Journal of Genetics. 12:101-109.

Heikkinen E., Lumme J. 1991. Sterility of male and female hybrids of Drosophila virilis and Drosophila lummei. Heredity. 67:1.

Hey J., Nielsen R. 2004. Multilocus Methods for Estimating Population Sizes, Migration Rates and Divergence Time, With Applications to the Divergence of Drosophila pseudoobscura and D. persimilis. Genetics. 167:747-760. 
Jha A.P., Rahman S.M. 1973. On crossing between Drosophila bipectinata and Drosophila malerkotliana. Cytologia (Tokyo). 38:425-436.

Johnson D.L.E. 1985. Genetic differentiation in the Drosophila athabasca complex. Evolution. 39:467-472.

Kearse M., Moir R., Wilson A., Stones-Havas S., Cheung M., Sturrock S., Buxton S., Cooper A., Markowitz S., Duran C., Thierer T., Ashton B., Meintjes P., Drummond A. 2012. Geneious Basic: An integrated and extendable desktop software platform for the organization and analysis of sequence data. Bioinformatics. 28:1647-1649.

Keightley P.D., Ness R.W., Halligan D.L., Haddrill P.R. 2014. Estimation of the Spontaneous Mutation Rate per Nucleotide Site in a Drosophila melanogaster Full-Sib Family. Genetics. 196:313-320.

Keightley P.D., Trivedi U., Thomson M., Oliver F., Kumar S., Blaxter M.L. 2009. Analysis of the genome sequences of three Drosophila melanogaster spontaneous mutation accumulation lines. Genome Research. 19:1195-1201.

Leaché A.D., Fujita M.K. 2010. Bayesian species delimitation in West African forest geckos (Hemidactylus fasciatus). Proceedings of the Royal Society B: Biological Sciences. 277:3071-3077.

Leaché A.D., Zhu T., Rannala B., Yang Z. 2019. The Spectre of Too Many Species. Systematic Biology. 68:168-181.

Legrand D., Tenaillon M.I., Matyot P., Gerlach J., Lachaise D., Cariou M.-L. 2009. SpeciesWide Genetic Variation and Demographic History of Drosophila sechellia, a Species Lacking Population Structure. Genetics. 182:1197-1206.

van der Linde K., Houle D. 2008. A supertree analysis and literature review of the genus Drosophila and closely related genera (Diptera, Drosophilidae). Insect Systematics \& Evolution. 39:241-267.

Luo A., Ling C., Ho S.Y., Zhu C. 2018. Comparison of Methods for Molecular Species Delimitation across a Range of Speciation Scenarios. Systematic Biology.

Marques D.A., Meier J.I., Seehausen O. 2019. A Combinatorial View on Speciation and Adaptive Radiation. Trends in Ecology \& Evolution. 34:531-544.

Mayr E. 1963. Animal species and evolution. Belknap Press of Harvard University Press.

Noor M.A.F., Grams K.L., Bertucci L.A., Reiland J. 2001. Chromosomal inversions and the reproductive isolation of species. Proceedings of the National Academy of Sciences. 98:12084-12088.

Obbard D.J., Maclennan J., Kim K.-W., Rambaut A., O’Grady P.M., Jiggins F.M. 2012. Estimating Divergence Dates and Substitution Rates in the Drosophila Phylogeny. Molecular Biology and Evolution. 29:3459-3473.

O’Grady P.M., DeSalle R. 2018. Phylogeny of the Genus Drosophila. Genetics. 209:1-25.

Pascual M., Chapuis M.P., Mestres F., Balanyà J., Huey R.B., Gilchrist G.W., Serra L., Estoup A. 2007. Introduction history of Drosophila subobscura in the New World: a microsatellite-based survey using ABC methods. Molecular Ecology. 16:3069-3083.

de Queiroz K. 1998. The General Lineage Concept of Species, Species Criteria, and the Process of Speciation. In: Howard D.J., Berlocher S.H., editors. Endless Forms: Species and Speciation. Oxford University Press. p. 57-75.

de Queiroz K. 2007. Species Concepts and Species Delimitation. Systematic Biology. 56:879_ 886.

R Core Team. 2013. R: A Language and Environment for Statistical Computing. Vienna, Austria: R Foundation for Statistical Computing. 
Rannala B., Yang Z. 2003. Bayes estimation of species divergence times and ancestral population sizes using DNA sequences from multiple loci. Genetics. 164:1645-1656.

Rannala B., Yang Z. 2017. Efficient Bayesian species tree inference under the multispecies coalescent. Systematic biology. 66:823-842.

Reid N.M., Hird S.M., Brown J.M., Pelletier T.A., McVay J.D., Satler J.D., Carstens B.C. 2014. Poor Fit to the Multispecies Coalescent is Widely Detectable in Empirical Data. Systematic Biology. 63:322-333.

Revell L.J. 2012. phytools: an R package for phylogenetic comparative biology (and other things): phytools: R package. Methods in Ecology and Evolution. 3:217-223.

Rieseberg L.H. 2001. Chromosomal rearrangements and speciation. Trends in Ecology \& Evolution. 16:351-358.

Schrider D.R., Houle D., Lynch M., Hahn M.W. 2013. Rates and Genomic Consequences of Spontaneous Mutational Events in Drosophila melanogaster. Genetics. 194:937-954.

Silvestro D., Warnock R.C.M., Gavryushkina A., Stadler T. 2018. Closing the gap between palaeontological and neontological speciation and extinction rate estimates. Nature Communications. 9.

Simpson G.G. 1951. The Species Concept. Evolution. 5:285-298.

Smith G., Lohse K., Etges W.J., Ritchie M.G. 2012. Model-based comparisons of phylogeographic scenarios resolve the intraspecific divergence of cactophilic Drosophila mojavensis: Phylogeography of Drosophila mojavensis. Molecular Ecology. 21:32933307.

Smith M.L., Carstens B.C. 2018. Disentangling the process of speciation using machine learning. bioRxiv.

Sukumaran J., Holder M.T. 2010. DendroPy: a Python library for phylogenetic computing. Bioinformatics. 26:1569-1571.

Sukumaran J., Knowles L.L. 2017. Multispecies coalescent delimits structure, not species. Proceedings of the National Academy of Sciences. 114:1607-1612.

Sweigart A.L. 2010. The Genetics of Postmating, Prezygotic Reproductive Isolation Between Drosophila virilis and D. americana. Genetics. 184:401-410.

Tamura K. 2003. Temporal Patterns of Fruit Fly (Drosophila) Evolution Revealed by Mutation Clocks. Molecular Biology and Evolution. 21:36-44.

Taylor S.A., Larson E.L. 2019. Insights from genomes into the evolutionary importance and prevalence of hybridization in nature. Nature Ecology \& Evolution. 3:170-177.

Wall J.D., Andolfatto P., Przeworski M. 2002. Testing models of selection and demography in Drosophila simulans. Genetics. 162:203-216.

Watada M., Matsumoto M., Kondo M., Kimura M.T. 2011. Taxonomic study of the Drosophila auraria species complex (Diptera: Drosophilidae) with description of a new species: Drosophila auraria species complex. Entomological Science. 14:392-398.

Wiley E.O. 1978. The Evolutionary Species Concept Reconsidered. Systematic Zoology. 27:17.

Willis S.C. 2017. One species or four? Yes!...and, no. Or, arbitrary assignment of lineages to species obscures the diversification processes of Neotropical fishes. PLOS ONE. 12: 0172349.

Wolf J.B.W., Ellegren H. 2016. Making sense of genomic islands of differentiation in light of speciation. Nature Reviews Genetics. 18:87-100.

Wood S.N. 2011. Fast stable restricted maximum likelihood and marginal likelihood estimation of semiparametric generalized linear models. Journal of the Royal Statistical Society (B). 73:3-36. 
791 Yang Y., Hou Z.-C., Qian Y.-H., Kang H., Zeng Q.-T. 2012. Increasing the data size to accurately reconstruct the phylogenetic relationships between nine subgroups of the Drosophila melanogaster species group (Drosophilidae, Diptera). Molecular Phylogenetics and Evolution. 62:214-223. Zoology. 61:854-865.

Yang Z., Rannala B. 2014. Unguided Species Delimitation Using DNA Sequence Data from Multiple Loci. Molecular Biology and Evolution. 31:3125-3135.

Yi S., Bachtrog D., Charlesworth B. 2003. A survey of chromosomal and nucleotide sequence variation in Drosophila miranda. Genetics. 164:1369-1381.

Yukilevich R. 2012. Asymmetrical patterns of speciation uniquely support reinforcement in Drosophila: asymmetrical patterns of reinforcement speciation. Evolution. 66:14301446. 